\title{
Holistic Health Status Questionnaire: developing a measure from a Hong Kong Chinese population
}

\author{
Choi Wan Chan ${ }^{1}$, Frances Kam Yuet Wong ${ }^{1 *}$, Siu Ming Yeung ${ }^{1}$ and Fok Sum²
}

\begin{abstract}
Background: The increased prevalence of chronic diseases is a global health issue. Once chronic disease is diagnosed, individuals face lifelong healthcare treatments, and the disabilities and disturbances resulting from their illness will affect the whole person. A valid tool that can measure clients' holistic care needs is important to enable us to identify issues of concern and address them early to prevent further complications. This study aimed to develop and evaluate the psychometric properties of a scale measuring holistic health among chronically ill individuals.

Methods: The research was an instrument development and validation study using three samples of Hong Kong Chinese people. The first sample $(n=15)$ consisted of stroke survivors who had experienced disruption of their total being, and was used as a basis for the generation of scale items. In the second and third samples $(n=319, n=303)$, respondents with various chronic illnesses were assessed in order to estimate the psychometric properties of the scale. A total of 52 items were initially generated, and 7 items with a factor loading less than 0.3 were removed in the process, as substantiated by the literature and expert panel reviews.
\end{abstract}

Results: Exploratory factor analysis identified a 45-item, 8-factor Holistic Health Status Questionnaire (HHSQ) that could account for $56.38 \%$ of the variance. The HHSQ demonstrated content validity, acceptable internal consistency $(0.59-0.92)$ and satisfactory convergent validity from moderate to high correlation with similar constructs $(r \geq 0.46, p<0.01)$.

Conclusions: The HHSQ tapped into the relational experiences and connectedness among the bio-psychosocial-spiritual dimensions of a Chinese person with chronic disease, with acceptable psychometric properties.

Keywords: Holistic Health Status Questionnaire, Holism, Holistic health, Instrument development, Factor analysis, Hong Kong Chinese

\section{Background}

Chronic diseases account for approximately $60 \%$ of population mortality globally [1]. The World Health Organization projects that of 64 million people who will die in 2015, 41 million will die of a chronic disease [2]. In Hong Kong (HK), approximately $61 \%$ of total registered deaths in 2006 were attributed to chronic diseases, including heart diseases, stroke, respiratory diseases and cancer [2]. Over $70 \%$ of communitydwelling elderly people in HK suffer from at least one

\footnotetext{
* Correspondence: frances.wong@polyu.edu.hk

${ }^{1}$ School of Nursing, The Hong Kong Polytechnic University, Hung Hom, Kowloon, Hong Kong

Full list of author information is available at the end of the article
}

kind of chronic disease [3]. Chronic diseases of the circulatory system, respiratory system and neoplasm accounted for at least $32 \%$ of the total allocated health expenditure in Hong Kong during the 2012 financial year [2]. At the individual level, in the case of a chronic disease like stroke, almost $40 \%$ of survivors are left with at least moderate disability [4-6] and become highly dependent on caregivers for help in daily activities on a long-term basis $[7,8]$.

People who are chronically ill can experience an array of sudden loss of bodily functions [9-11]; psychological and emotional distress [12-14]; the loss of role and selfidentity [12] leading to significant disruption in individual, family and social life [15]; and concerns for the 
future, sustaining hope, ability to heal, and maintaining spiritual strength [16]. Individuals can be viewed as being in multi-faceted relationships, and illness can be considered as a disturbance to these relationships. These relationships involve the connections between body, mind and transcendence, the nexus of the ecological, physical, familial, social and political environments [17]. Hence, these relational disturbances/experiences typically allude to the fact that the bio-physical, psychological, social and spiritual dimensions are not separate from the experiences of an individual as a whole, when that individual is chronically ill $[18,19]$. Once chronic disease is diagnosed, individuals face lifelong healthcare treatments, and their disabilities and disturbances resulting from the illness fundamentally require long-term wholeperson healthcare support. Among the chronically ill groups, stroke survivors tend to have more identified negative effects in terms of physical impairment [20], as well as psychological and social impacts $[15,21,22]$. A Danish study reported that $23.6 \%$ of stroke patients purchased antidepressants during follow-up, a much higher rate than in the comparison osteoarthritis group, which documented a rate of $9.1 \%$ [23]. Holistic healthcare attends to all the disturbed relationships of an ill person as a whole, even though the person is not thereby completely resilient to perfect wholeness. A holistic framework enabling healthcare to address all aspects of bio-physical, psychological, social and spiritual support in the management of chronic diseases is obviously needed.

The fundamental dimensions of holism encompass physical, psychological, social and spiritual aspects [24]. Holism in nursing "involves identifying the interrelationships of the bio-psycho-social-spiritual dimensions of a person" [25]. These aspects of holism essentially provide a generic framework for addressing the holistic well-being and concerns experienced by individuals with chronic disease during their long-term care.

To enhance holistic healthcare for chronic diseases in a Chinese population, the cultural attributes that underpin the generic holistic framework appear to be underexplored. Culturally, the concept of holism in Chinese societies is derived from the integrated beliefs of Confucianism, Buddhism and Taoism [26-29], which assert the importance of maintaining a balanced and holistic mind-body state. The other attribute of holism in Chinese culture is that the individual self is highly related to the family context. Close interpersonal relationships such as the emotional bond with family members are salient in Chinese culture [30]. Family connectedness becomes especially important when the family assumes an important role in taking care of a member who is experiencing illness. During the course of illness, Chinese traditionally believe that health and the associated interpersonal outcomes are related to fate, transcendent forces or the predestiny of the virtuous and evil-doers, with strong psychological feelings of guilt and self-blame [29, 31].

As holistic healthcare for chronic disease requires attention to the bio-psycho-socio-spiritual disturbances and concerns of individuals, addressing and assessing holistic well-being and concerns experienced by the individual is where the task begins. Research has been conducted to develop and test holistic health measures [32-34], but very little has focused on the Chinese population with chronic illnesses. Researchers embarking on the development of holistic health measures share some common world views. Existing well-established health status instruments such as the SF Quality of Life measures view health as compartmentalized into physical, mental, social and spiritual dimensions, rather than as a holistic entity [35]. Holistic health researchers assume that respondents perceive all aspects of the self as interrelated, thus expressing the self as a gestalt [36]. The expressions are subjective, so the use of the language in the items should be grounded in the experience of these individuals and reflect their responses. The generation of items for holistic measures therefore often begins with qualitative interviews to capture the stakeholders' perspectives and descriptions in the experience of their health state [35, 37]. During this process, clinicians provide comments on the components, which help validate the statements [36]. The instrument is then subjected to reliability and validity testing.

There is a dearth of valid instrumentation addressing holistic health and well-being, particularly among Chinese people with chronic diseases. For health status measurements designed and tested in Western culture(s), difficulties were encountered in producing corresponding cultural and linguistic expressions in Chinese equivalent to the item wordings $[38,39]$. Similarly, differences in health status measurement results between Western and Eastern cultures are widely recognized [38-42]. Embedded differences in cross-cultural interpretations include health status measurement domains, particularly those related to social functioning [38, 42], mental health [39, 42], physical functioning [39], and vitality, for measuring both physical and mental energy as well as fatigue [38, 39, 42]. In addition, for generic health status measurements, others have suggested that the domain of family functioning might be needed to acknowledge the impact of health on family life in cultures in which family life might play a more central role in individuals' lives [39, 42]; the domain for spiritual aspects also appears lacking [43]. In view of the gaps in instrumentation, the purpose of this study was to develop an instrument with culturally-sensitive attributes that tap into Chinese people's holistic health and wellbeing concerns in relation to chronic diseases. The 
conceptual framework of bio-psycho-social-spiritual holism was used to guide the present study.

\section{Methods}

This research was a methodological study used to assess the validity (content/construct validity) and reliability of an instrument [44]. The study consisted of two stages. The first pertained to scale development, including the generation of scale items and the establishment of the content validity of the scale. The second phase aimed to establish the psychometric properties of the Holistic Health Status Questionnaire (HHSQ) that was developed in the first stage. The psychometric analysis involved construct validity and reliability in terms of evaluating the internal consistency of the scale using Cronbach's $\alpha$ coefficients. The construct validity included a factor analysis and convergent validity. In convergent validity, it was anticipated that the constructs identified in HHSQ would be correlated with the SF-12 Health Survey (a quality of life measurement) based on the premise that an individual with a higher level of holistic health status is expected to enjoy a better quality of life.

\section{Stage 1: development of the HHSQ}

The items of the HHSQ were first generated among post-stroke patients involved in a randomized controlled trial that included the measurement of holistic wellbeing as one of the outcomes. Stroke survivors were selected as the target group for item generation because they tend to experience all of the potential impacts of a chronic illness, including physical [20], psychological [23], social [21] and spiritual [9] dimensions. Semistructured, face-to-face individual interviews with 15 discharged post-stroke patients were conducted to explore the holistic concerns of these patients within the first week of returning home after an acute episode involving a physical consult. The participants were selected by a purposive sampling method based on the following inclusion criteria: (1) Chinese, (2) aged >18 years, (3) first stroke, (4) cognitive status assessed by the MiniMental Status Examination with scores $>21$ [45], (5) able to communicate, (6) no debilitating co-morbidity, and (7) discharged home. Patients diagnosed with Transient Ischemic Attacks or with co-existing mental disorders were excluded from the study. This sample consisted of participants recruited from the stroke unit of a general hospital in Hong Kong, with ages ranging from 53 to 79 (mean $(M)=64.7$, standard deviation $(S D)=8.9$ ); seven were male and eight were female. All had received education at primary level or below, and $33.3 \%$ described themselves as having a religious background, such as Buddhism (26.7\%) or Christianity (6.6\%). Almost all were supported by family caregivers. The average length of hospitalization was 13 days. All informants experienced residual problems, such as limb weakness, memory loss, dizziness, fatigue and sleep disturbances. During the interview, the start-up interview question "Would you please describe the event(s) directly connected with your stroke experience?" was asked, and follow-up questions were then posed to enable further exploration of participants' initial answers [46]. The interviews were audiotaped and transcribed by a research assistant, and the accuracy of the transcripts was checked by a research team investigator. Data saturation was achieved when no information could be identified that added to new content units or themes [46]. The research team investigator analyzed all the qualitative data using content analysis. Units of content carrying the same meaning were crosschecked, discussed and eventually collapsed into themes, with consensus achieved with the other three research team investigators in multiple meetings. A total of 51 items representing the five aspects of concern in the physical, psychological, social, spiritual and cultural domains emerged from the data. These items were then reviewed by a panel of 5 experts including 1 nurse manager (medical), 1 specialty nurse (stroke), 1 community nurse, 1 holistic care educator and 1 researcher in the field of postdischarge and/or stroke care. The content validity index (CVI) was used to rate the content relevance of the items on a four-point scale: $1=$ completely irrelevant, $2=$ not relevant, $3=$ relevant, and $4=$ completely relevant. The experts reviewed the items independently. The CVI was calculated using the percentage of total items rated by the experts as either 3 or 4 , a CVI rating above 0.8 being considered valid [47]. Besides the rating, the experts suggested rewording the sentences in 8 of the items to make the sentences more comprehensible. Apart from the 51 items, one of the panel experts suggested adding a new item, 'I feel pain all over my body', which was observed to be a common complaint in clinical encounters. The research team revisited the data and found that this complaint of pain all over the body had been mentioned by a number of informants but subsumed under the overall discomfort item. After discussion and considering the clinical reality, this item of 'pain all over the body' was included as one of the items. The revised version of the questionnaire was again sent to the expert panel for review. All scale items were judged as content valid with a score of either 3 or 4 in the second round of the expert review.

\section{Stage 2: psychometric properties of the HHSQ}

The HHSQ developed in Stage 1 generated items that contained direct expressions of holistic concern after 
an episode of illness. Interestingly, these items conveyed no specific description of the illness concerned. The research team then asked if the HHSQ would be applicable to a population with residual health concerns other than stroke. We therefore selected two samples with known chronic illnesses for further testing.

\section{Participants}

Two independent samples of participants with chronic illnesses were recruited to establish the psychometric properties of the HHSQ. Sample 1 reported chronic illnesses, but no specific illness type was used to identify the factor structure and establish the scale reliability. Subjects were adults recruited from general health or social service settings. In sample 2, we deliberately chose patients from 3 chronic disease groups who were regularly followed up in the daycare service centers or hospital outpatient departments. These groups of chronically ill patients were used to evaluate the relationship between SF12 and HHSQ in testing the convergent validity of the instrument. The research assistant read the items to the respondents and helped them fill in the questionnaire, so the literacy of the respondents was not a concern in data collection. The instrument took 30 min to complete. A minimum of 260 subjects was considered adequate based on the recommended requirement of at least 5 participants per item for the psychometric assessment of an instrument [48].

Sample 1: identify factor structure and estimate scale reliability A convenience sample of 319 adult Chinese participants recruited from a regional cluster of healthcare settings, including a regional hospital $(22.0 \%)$, a day rehabilitation center (6.0\%), a nursing home (20.3\%), and an elderly center (51.7\%), was used to identify the scale factor structure and establish the internal consistency reliability. A total of 345 subjects were approached, resulting in a response rate of $92.6 \%$. The sample age ranged from 21 to 99 years $(M=76.7$, $\mathrm{SD}=10.4$ ), and $67.7 \%$ were female. All participants had reported chronic illnesses, with $60.5 \%$ having $\geq 2$ types of chronic disease, such as stroke, coronary disease, diabetes, hypertension, renal failure or chronic obstructive pulmonary disease (COPD). The majority (72.3\%) had primary level education, and $71.8 \%$ reported having a religious background.

Sample 2: perform convergent validity A convenience sample of 303 adult Chinese participants with specific chronic diseases including COPD $(n=91,30.0 \%)$, diabetes $(n=110,36.3 \%)$, and chronic renal failure $(n=102,33.7 \%)$ was recruited from a medical unit of a regional hospital (5.0\%), daycare service centers $(58.7 \%)$ and a hospital outpatient department (36.3\%).
Three hundred and eight subjects were approached, resulting in a response rate of $98.4 \%$. The sample age ranged from 18 to 91 years $(\mathrm{M}=61.0, \mathrm{SD}=13.1)$, and $37.6 \%$ were female. More than half of the sample (56.8 \%) had secondary level education, and the majority $(71.8 \%)$ reported having a religious background.

\section{Procedures}

Ethical approval to conduct the study was obtained from the Hong Kong Polytechnic University Human Subjects Ethics Subcommittee and Joint Chinese University of Hong Kong-New Territories East Cluster Clinical Research Ethics Committee. The study's nature and purpose were explained to the participants by a research team member. They were assured of their privacy and anonymity. Each participant who agreed to take part in the study was asked to sign an informed consent, complete the HHSQ, HK-specific SF-12 and provide demographic information and the history of their chronic disease.

\section{Measures \\ The HHSQ}

After expert review for content validity, the initial HHSQ consisted of 52 items. Each item was rated on a 4-point scale ranging from "none of the time" to "all of the time". The HHSQ scores were obtained by summing the item scores. A higher score indicated better holistic well-being.

\section{The SF-12 Health Survey}

The SF-12 Health Survey [49] was developed as a shorter version of the SF-36 Health Survey. The SF-12 consists of 12 items in two domains: the physical and mental component summaries (PCS and MCS). The 12 items include two from each of the Physical Functioning, Role-Physical, Role-Emotional and Mental Health subscales, and one from each of the Bodily Pain, General Health, Vitality and Social Functioning subscales of the SF-36. The original SF-12 Health Survey has been translated, validated and shown to be reliable for use in a HK Chinese population $[50,51]$. A higher score indicates better quality of life on the SF-12.

\section{Statistical analyses}

Data were analyzed using the IBM SPSS Statistics software, version 20. Descriptive statistics were used to summarize the demographic data. An exploratory factor analysis (EFA) using principal component analysis (PCA) and varimax rotation was performed to examine the factor structure of the HHSQ. A Kaiser-Meyer-Olkin $(\mathrm{KMO})$ score $>0.6$, which indicates adequate sample size for the factor analysis, and a significant value for Bartlett's test of sphericity were employed to determine the factorability of the data [52]. The scree plot analysis 
and Kaiser-Guttman criterion with eigenvalues greater than 1.0 as the criteria were used to extract factors. Factor loadings that exceeded the criterion of 0.30 were used as a cut-off point to retain the significant items [53]. The internal consistency of the HHSQ was assessed through Cronbach's $\alpha$ coefficients, with a value greater than 0.60 considered acceptable for a newly-developed instrument [54]. For convergent validity, Pearson's correlation was used. A 0.05 level of significance was employed.

\section{Results}

\section{Exploratory factor analysis}

The significant value of Bartlett's test of sphericity $(p<.001)$ and the KMO value of 0.89 indicated that the assumptions for factor analysis were met. Initial exploratory factor analysis using eigenvalues greater than 1.0 yielded 12 factors, which accounted for $61.11 \%$ of the total explained variance. Based on the scree plot, a discontinuity of the steep slope was around 7 to 9 factors, where the 'elbow' of the scree plot was located in this leveled-off area. From factor 7 to factor 12, the eigenvalues were 1.47, $1.30,1.16,1.13,1.08,1.06$, respectively. It was decided to extract an eight-factor structure model, as this provided the best interpretation of items in terms of congruency and relevancy, as well as contributing a reasonable total variance (8-factor $52.59 \%$ versus 7 -factor $50.09 \%$ and 9-factor $54.83 \%$ ). When examining the 8factor model, item deletion was executed based on a number of considerations. These items were below the factor loading cut-off criterion of 0.3 , with communalities $<0.20$, and with multiple loadings. We also examined the item-domain correlations to determine whether the Cronbach's alpha changed with the removal of the item. The research team then considered the conceptual congruence of the item with the factors. Finally, seven items were removed from the initial 52item HHSQ. One item, 'I don't get good sleep', was retained in spite of a low factor loading (0.23) under the factor of physical symptoms, as sleeplessness had emerged from the qualitative data as a common and frequent concern and was conceptually relevant to the factor characteristic. The factor analysis was re-run after item deletion.

The final 8-factor, 45-item HHSQ accounted for $56.38 \%$ of total variance (Table 1 ). The 8 factors were (1) psychological expression (12 items, item factor loadings ranging from 0.54 to 0.72 ), (2) changes in self and family (seven items, 0.40-0.74), (3) physical symptoms (ten items, 0.27-0.63), (4) social and family connectedness (three items, 0.51-0.85), (5) fatalism (three items, $0.78-0.88)$, (6) religion and faith (three items, 0.32-0.90), (7) self-query (four items, $0.45-0.63$ ), and (8) coping style (three items, 0.63-0.83). The final eigenvalues (and variances) of the factors were 11.46 (25.48 \%), 3.37 (7.50 \%), 2.29 (5.09\%), 2.16 (4.81\%), 2.04 (4.52\%), 1.44 (3.21\%), 1.40 (3.10\%), and 1.21 (2.68\%), respectively. Based on the conceptual relevancy of the factor, the item 'I don't get good sleep' in the final model achieved a factor loading of 0.27 , close to the set criterion, and thus remained in factor 3-physical symptoms, as initially decided. There were 18 complex items carrying a factor loading that would allow them to be placed in more than one factor. Thirteen of these items were placed in the factors where they had the highest loadings. The remaining five items were housed in the host factors where they fit conceptually and bore a reasonable loading. These items included 'I feel that my illness is causing trouble to my family' (factor loading 0.40) under the factor of changes in self and family; 'There is hope in my future' (0.32) under religion and faith; 'I ask, "Why do I have this illness?"' (0.45) under self-query; and "There is something wrong with my body' (0.36) and 'I feel weak in my body/limbs' (0.42) under physical symptoms.

\section{Reliability}

The subscales' $\alpha$ from factor 1 to factor 8 were 0.92 , $0.82,0.81,0.73,0.88,0.64,0.62$, and 0.59 respectively. The item-to-subscale correlations were $0.58-0.75$ (factor 1), 0.49-0.62 (factor 2), 0.31-0.64 (factor 3), 0.30-0.73 (factor 4), 0.67-0.84 (factor 5), 0.17-0.66 (factor 6), 0.27-0.47 (factor 7), and 0.34-0.50 (factor 8) (Table 2).

\section{Convergent validity}

Significant and moderate correlations were found between the total HHSQ scores and the scores of the two domains of the SF-12: the Physical Component Summary, PCS $(0.46, p<0.01)$ and the Mental Component Summary, MCS $(0.70, p<0.01)$. Significant but weak correlations (0.2) were also found between all of the subscale scores of the HHSQ and the domains of SF-12 (Table 3), except between the subscale 'social and family connectedness' and PCS $(0.06, p>0.05)$.

\section{Discussion}

The purpose of this study was to develop and evaluate the psychometric properties of the HHSQ. Findings yielded an initial factor structure of the HHSQ through EFA with acceptable internal consistency and satisfactory convergent validity. The lack of a tool for assessing holistic health using individuals' direct expressions highlights an important gap in the research in this area. This initial development of the HHSQ is the first step in the task of filling this gap, which has cultural and linguistic relevance. It is a promising tool for use after future scale refinement and validation.

The HHSQ consists of the domains of psychological expression, changes in self and family, physical symptoms, 
Table 1 Rotated component matrix of the 45-item, 8-factor Holistic Health Status Questionnaire

\begin{tabular}{|c|c|c|c|c|c|c|c|c|}
\hline \multirow[t]{2}{*}{ Item } & \multicolumn{8}{|l|}{ Factor } \\
\hline & 1 & 2 & 3 & 4 & 5 & 6 & 7 & 8 \\
\hline My mood is delighted. & .723 & .174 & .040 & .405 & .038 & .074 & .030 & .000 \\
\hline I feel happy. & .683 & .178 & .004 & .346 & .045 & .144 & .017 & .054 \\
\hline I feel very frightened. & .682 & .045 & .200 & .107 & .074 & .058 & .009 & .023 \\
\hline I feel confused. & .669 & .157 & .313 & .116 & .134 & .072 & .208 & .021 \\
\hline With this illness, I cannot take care of myself. & .664 & .196 & .079 & .001 & .058 & .071 & .086 & .009 \\
\hline My heart feels heavy. & .659 & .134 & .251 & .198 & .119 & .043 & .096 & .023 \\
\hline I feel sad. & .657 & .151 & .260 & .206 & .121 & .098 & .253 & .019 \\
\hline I am really afraid that my illness will get worse. & .617 & .138 & .227 & .017 & .057 & .071 & .155 & .032 \\
\hline I feel really uncomfortable emotionally. & $\underline{.595}$ & .213 & .190 & .216 & .129 & .052 & .263 & .067 \\
\hline I have peace in my heart. & .592 & .107 & .083 & .430 & .026 & .037 & .041 & .138 \\
\hline I feel that my illness is causing trouble to my family. & .566 & .397 & .037 & .221 & .183 & .094 & .026 & .016 \\
\hline Living with this illness is hard. &.$\underline{.563}$ & .339 & .222 & .132 & .201 & .066 & .031 & .064 \\
\hline I want to cry. &.$\underline{.535}$ & .059 & .262 & .140 & .084 & .180 & .124 & .076 \\
\hline There is something wrong with my body. & .517 & .053 & .363 & .056 & .008 & .088 & .151 & .130 \\
\hline I ask, "Why do I have this illness?" & .507 & .097 & .106 & .079 & .109 & .078 & .446 & .015 \\
\hline I feel weak in my body / limbs. & .434 & .220 & .415 & .017 & .042 & .093 & .026 & .023 \\
\hline It is not the same any more at work. & .134 & .739 & .184 & .019 & .076 & .101 & .058 & .010 \\
\hline I feel different than before when at home. & .180 & .669 & .153 & .159 & .073 & .109 & .083 & .012 \\
\hline With this illness, I feel I have lost all my freedom. & .345 & .637 & .007 & .112 & .081 & .034 & .176 & .021 \\
\hline I have become more clumsy than before. & .166 & .612 & .335 & .031 & .165 & .033 & .141 & .129 \\
\hline I do not go out as often as before. & .309 & .593 & .002 & .027 & .140 & .039 & .212 & .057 \\
\hline It is a burden for my family to take care of me. & .416 & .467 & .147 & .369 & .081 & .043 & .079 & .104 \\
\hline I feel dizzy. & .285 & .037 & .628 & .044 & .010 & .043 & .055 & .161 \\
\hline I feel pain all over my body. & .264 & .013 & .596 & .132 & .073 & .001 & .060 & .027 \\
\hline My appetite has worsened. & .021 & .384 & .533 & .014 & .057 & .036 & .239 & .061 \\
\hline I feel uncomfortable all over my body. & .483 & .161 & .531 & .023 & .089 & .042 & .108 & .041 \\
\hline I feel really tired. & .315 & .244 & .526 & .043 & .055 & .184 & .031 & .112 \\
\hline My memory has worsened. & .142 & .130 & .516 & .144 & .177 & .014 & .254 & .109 \\
\hline I get headaches. & .385 & .086 & .480 & .119 & .031 & .056 & .189 & .125 \\
\hline My family gives me great comfort. & .131 & .102 & .195 & .848 & .013 & .050 & .025 & .009 \\
\hline My family cares a lot about me. & .131 & .085 & .141 & .834 & .004 & .044 & .010 & .093 \\
\hline My friends give me great comfort. & .165 & .192 & .060 &.$\underline{.511}$ & .074 & .037 & .048 & .002 \\
\hline There is hope in my future! & .007 & .102 & .312 & .346 & .231 &.$\underline{323}$ & .180 & .057 \\
\hline I believe that it is predestined! & .151 & .093 & .048 & .026 & .880 & .050 & .138 & .096 \\
\hline I believe it is fate. & .171 & .068 & .011 & .024 & .861 & .039 & .117 & .044 \\
\hline I think this is divine intervention. & .113 & .058 & .035 & .028 & .775 & .044 & .173 & .055 \\
\hline I believe the Heavens/God/my religion is taking care of me. & .109 & .052 & .071 & .091 & .075 & .895 & .096 & .037 \\
\hline I find it helpful to pray to God/gods/my religion. & .096 & .084 & .116 & .048 & .090 & .884 & .031 & .013 \\
\hline I've caused my own illness! & .221 & .144 & .058 & .064 & .233 & .078 & .625 & .041 \\
\hline I still have much unfinished business. & .333 & .184 & .014 & .064 & .038 & .074 & .549 & .112 \\
\hline I believe that this is karma! & .008 & .015 & .064 & .068 & .296 & .143 & .517 & .011 \\
\hline I don't get good sleep. & .100 & .151 & .272 & .238 & .028 & .155 & .287 & .236 \\
\hline So I am sick, anyway I have to accept it. & .032 & .008 & .066 & .053 & .041 & .071 & .085 & .830 \\
\hline
\end{tabular}


Table 1 Rotated component matrix of the 45-item, 8-factor Holistic Health Status Questionnaire (Continued)

\begin{tabular}{|c|c|c|c|c|c|c|c|c|}
\hline I can't help getting sick, so I should not think too much about it. & .108 & .089 & .054 & .048 & .049 & .181 & .134 & .685 \\
\hline I think, "Let it be!" & .087 & .002 & .000 & .091 & .186 & .098 & .130 & .634 \\
\hline Eigenvalues & 11.46 & 3.37 & 2.29 & 2.16 & 2.04 & 1.44 & 1.40 & 1.21 \\
\hline$\%$ of variance by factor & 25.48 & 7.50 & 5.09 & 4.81 & 4.52 & 3.21 & 3.10 & 2.68 \\
\hline
\end{tabular}

Items of respective factors with factor loadings bold and underlined. 1 psychological expression, 2 changes in self and family, 3 physical symptoms, 4 social and family connectedness, 5 fatalism, 6 religion and faith, 7 self-query, 8 coping style

social and family connectedness, fatalism, religion and faith, self-query, and coping style. It contains not only the bio-psycho-social and spiritual aspects as denoted in the generic frame of holism, but is also characterized by elements in relation to chronic illness, featuring Chinese cultural attributes and expressions. Though the sample subjects in this study are of Chinese origin, the attributes and expressions are congruent with the literature to be discussed below, which includes populations other than the Chinese.

Spirituality leads to finding a purpose and meaning in life that is attributed to human existence [55]. Being disabled after an illness is a traumatic event, and the phenomenon of searching for meaning after a critical event is commonly reported in both Eastern and Western literature [24, 56-58]. Individuals who were chronically ill often raised questions about their own identities in their life stance, trying to find answers and seek some purpose in what happened to change their health. Individuals sometimes interpreted the meaning of their experience in relation to nature, fate or a higher power [57, 59]. This is consistent with our study findings, in which individuals became fatalistic, relating/turning to religion and seeking a sense of hope when faced with the harsh reality of being disabled [60-62]. Items such as 'I believe it is fate,' 'I believe that it is predestined', 'I find it helpful to pray to God/gods/my religion' and 'There is hope in my future' were the expressions that demonstrated this phenomenon.

The integrated beliefs of Confucianism, Buddhism and Taoism inspire the concept of holism in Chinese societies [26-29]. An individual self is constructed in webs of relationships, as found in the Confucian 'five basic relationships' tradition $[26,63]$. Three out of these five basic relationships occur within the family: fatherson, husband-wife, and elder-younger. The individual is traditionally held with close relatedness within the family, where the holism (wholeness) of an individual in Chinese culture is contextualized. Inner and deep feelings can only be shared with those with whom a person has close ties [64]; in particular, the emotional bond with family members is salient [30]. The importance of family connectedness and the important cultural roles assumed by Chinese families in taking care of sick members, as reported in this sample of HK Chinese, demonstrate the relatedness of the individual with the family during the course of illness, which underpins the concept of wholeness of an individual. Items reported from our sample, such as 'My family gives me great comfort' and 'My family cares a lot about me', reflect the importance of this aspect.

The styles of coping and living with chronic diseases among the HK Chinese participants were shaped by traditional Chinese values and beliefs. The attitude of 'letting go' was one of the coping styles. Although 'letting go' is a common response found in both eastern and western literature [16, 27, 65], the meaning of 'letting go' among Chinese is derived from Confucian, Buddhist and Taoist beliefs, namely that illness is part of life, and over-attachment to an illness event will lead to suffering. Freedom from emotional turmoil and maintaining peace, balance and harmony to keep goodness in mind-body-spirit in order to be more resilient in the

Table 2 Reliability statistics

\begin{tabular}{lccc}
\hline Factor & Cronbach's a & Item-to-subscale correlation & Subscale-to-total correlation \\
\hline 1 Psychological expression & 0.92 & $0.58-0.75$ & 0.92 \\
2 Changes in self and family & 0.82 & $0.49-0.62$ & 0.73 \\
3 Physical symptoms & 0.81 & $0.31-0.64$ & 0.80 \\
4 Social and family connectedness & 0.73 & $0.30-0.73$ & 0.39 \\
5 Fatalism & 0.88 & $0.67-0.84$ & 0.39 \\
6 Religion and faith & 0.64 & $0.17-0.66$ & 0.02 \\
7 Self-query & 0.62 & $0.27-0.47$ & 0.59 \\
8 Coping style & 0.59 & $0.34-0.50$ & 0.12 \\
\hline
\end{tabular}


Table 3 Pearson correlations of 8-factor Holistic Health Status Questionnaire with SF-12 Health Survey

\begin{tabular}{|c|c|c|c|c|c|c|c|c|c|}
\hline & \multicolumn{8}{|c|}{ HHS components } & \multirow[b]{2}{*}{$\mathrm{HHS}$} \\
\hline & $\begin{array}{l}\text { Psychological } \\
\text { expression }\end{array}$ & $\begin{array}{l}\text { Physical } \\
\text { symptoms }\end{array}$ & $\begin{array}{l}\text { Changes in self } \\
\text { and family }\end{array}$ & Fatalism & $\begin{array}{l}\text { Social and family } \\
\text { connectedness }\end{array}$ & $\begin{array}{l}\text { Self- } \\
\text { query }\end{array}$ & $\begin{array}{l}\text { Religion } \\
\text { and faith }\end{array}$ & $\begin{array}{l}\text { Coping } \\
\text { style }\end{array}$ & \\
\hline \multicolumn{10}{|l|}{ HHS components } \\
\hline \multicolumn{10}{|l|}{$\begin{array}{l}\text { Psychological } \\
\text { expression }\end{array}$} \\
\hline Physical symptoms & $.686^{* *}$ & & & & & & & & \\
\hline $\begin{array}{l}\text { Changes in self and } \\
\text { family }\end{array}$ & $.681^{* *}$ & $.716^{* *}$ & & & & & & & \\
\hline Fatalism & $.208^{* *}$ & $.270^{* *}$ & $.258^{* *}$ & & & & & & \\
\hline $\begin{array}{l}\text { Social and family } \\
\text { connectedness }\end{array}$ & $.243^{* *}$ & $.157^{* *}$ & .058 & .074 & & & & & \\
\hline Self-query & $.330^{* *}$ & $.229^{* *}$ & $.253^{* *}$ & $.157^{* *}$ & .029 & & & & \\
\hline Religion and faith & $.173^{* *}$ & .078 & $.206^{* *}$ & .077 & $.233^{* *}$ & .034 & & & \\
\hline Chinese coping skills & $.253^{* *}$ & $.125^{*}$ & .016 & $.126^{*}$ & $.257^{* *}$ & $.125^{*}$ & .090 & & \\
\hline HHS & $.899^{* *}$ & $.859^{* *}$ & $.836^{* *}$ & $.366^{* *}$ & $.287^{* *}$ & $.395^{* *}$ & $.267^{* *}$ & $.245^{* *}$ & \\
\hline \multicolumn{10}{|l|}{ SF12 } \\
\hline PCS & $.258^{* *}$ & $.533^{* *}$ & $.583^{* *}$ & $.173^{* *}$ & .057 & $.118^{*}$ & $.126^{*}$ & $.116^{*}$ & $.464^{* *}$ \\
\hline MCS & $.671^{* *}$ & $.558^{* *}$ & $.559^{* *}$ & $.239^{* *}$ & $.247^{* *}$ & $.237^{* *}$ & $.177^{* *}$ & $.231^{* *}$ & $.698^{* *}$ \\
\hline
\end{tabular}

course of suffering could transcend to a coping attitude of non-action, which is valued in Taoism $[26,58,66]$. As such, Chinese participants might exhibit a 'trying not to think too much' attitude (a way of being unconcerned) and try to learn to live with their reality. The coping style of accepting reality could possibly be interpreted as a passive acceptance of reality when circumstances are beyond their personal control or when they are under physical constraints such as living with permanent physical disabilities. The individuals had not actually taken active measures to solve the problem or make changes. This might account for our study finding that those with higher scores of coping style in the HHSQ had lower SF-12 Health Survey PCS (physical component summary) scores. The study by Siu et al. [67] also revealed that the Confucian virtue of forbearance may have negative consequences for health and well-being, with passive adaptive coping (i.e. accepting reality, letting fate have its way) correlated with more reported physical and behavioral symptoms.

It has been discussed that the same data, after extraction with several different techniques and followed by varimax rotation, resulted in similarities among factor solutions. The choice between using principal component analysis (PCA) and principal factor analysis (PFA) depends on the assessment of the fit between models, the data set, and the goals of the research [52]. The approach employed in this study was sensible, based on the premise that an EFA using
PCA and varimax rotation yielded factors that were congruent with the conceptual understanding of holistic health. The final factor model explaining $56.38 \%$ of the variance was considered acceptable [68]. If an item did not effectively measure the factor of interest, it should be removed to improve the factor content validity $[52,69]$. Seven items were removed from the factor analysis as they were less congruent with the factor content or marker items of their respective factors. Almost all items in the final model were significantly loaded to a factor (factor loading of 0.3 or above) [69, 70], indicating that these items were effective indicators representing the interest of their respective factors.

The internal consistency indicated acceptable values according to the set criterion for a newly-developed instrument. Although item-to-subscale correlations above 0.3 are usually considered good [71], others have suggested that the item-to-subscale correlation can be above 0.2 [72] or even 0.15 [73]. The findings of item-tosubscale correlations in the present study were considered acceptable. Of the 45 items, only one, 'There is hope in my future!, under factor 6 'religion and faith', obtained a low correlation (0.17) and might require further exploration in future scale validation or refinement. Though the subscale-to-total correlations, factor 6 'religion and faith', and factor 8 'coping style' had relatively low correlations, the findings revealed that each of them contained three items with significant factor loadings 
that are generally needed $[68,70,74]$. It is plausible to expect items to be less homogenous within a three-item domain because the stability of a domain can be caused by an increase in the number of significant items, reflecting the depth and breadth dimensions of the domain. However, Factors 6,7 and 8 had an acceptable mean inter-item correlation. When there are a small number of items in the domain, it may be better to report the mean inter-item correlation for the items regarding the domain homogeneity, and an optimal mean inter-item correlation values range from 0.2 to 0.4 is recommended [75]. Factors 6,7 and 8 had a mean inter-item correlation of $0.38,0.29$ and 0.33 , respectively. Based on the Chinese cultural and conceptual perspectives, these factors were relevant for retention in the model. As an increase in the number of significant items reflecting the depth and breadth dimensions of a domain improves factor clarity or stability as well as enhancing subscale-to-total correlations, the domains of religious beliefs and coping style among Hong Kong Chinese people need to be further explored for future scale refinement.

Although factor analysis plays a unique role in scale development, the subjective and judgmental nature of decisions made during the analysis process is often the basis for serious criticism, thus prior knowledge about the research area is crucial in the process [44]. The present study, based on understanding from the literature and expert input from health professionals, and informed by the findings of the qualitative study, generated items that were subjected to testing for factor analysis. Conservatively setting the factor loading at a minimal 0.30 as a significant criterion level prevented the inappropriate dropping of items or factors in the early stage of scale development using exploratory factor analysis. The conceptual guide and qualitative findings helped further support the decisions during the factor analysis procedures. Given that the first factor, accounting for $25 \%$ of the variance, represented chiefly the psychological dimension, we decided to include factors of lesser variance as they contained the bio-psycho-social and spiritual aspects as denoted in the generic frame of holism, and these factors were consistent with the Chinese cultural and conceptual perspectives. Undoubtedly, further studies with larger sample sizes are needed to improve the depth and breadth dimensions for the factors with smaller variances.

The optimal holistic health and well-being of an individual are aligned with their experiencing a better quality of life. Satisfactory construct validity of the HHSQ was found in the convergent validity, with a significant correlation between the overall HHSQ and the two main domains, PCS and MCS, of the quality of life measure, the SF-12 Health Survey. It should be noted that weak relationships were found between some individual factors of the HHSQ and the quality of life measure. The measure of holistic health, however, unlike SF-12, does not measure the compartmentalized dimensions of the physical and mental domain. Rather, the HHSQ aims at reporting the respondents' state of health using descriptions that are grounded in their lived experiences and world view [35-37].

It is interesting to note that the statements generated from the respondents in this study corresponded with those reported in the study by Faull [36] conducted in New Zealand. Both groups expressed holistic health concerns in terms of one's relationship with oneself, one's family, the Higher Being and healthy functioning $[36,76]$. In this study, however, the Chinese respondents tended to suggest perspectives that were related to fate, letting go and passive acceptance. These measures have been described as a uniquely Chinese way of coping in yielding to predestiny (feng-shui) and serendipity (yuan-fen) [67]. The HHSQ has proven to be an instrument with established psychometric properties that help in understanding Chinese perceptions of holistic health, particularly for those with chronic illnesses.

\section{Limitations and implications for the research}

The present study has limitations. While taking account of patients' health conditions and the feasibility of recruiting participants in a sufficiently large sample, this study was limited to recruiting a proportionate sample of participants with different kinds of chronic diseases, although efforts were made to perform wide-scale recruitment of participants who were all chronically ill and required holistic care. It was a drawback that the subjects used in this study to develop and evaluate the scale were very different in many ways in terms of religious background, type and stage of chronic disease, and health condition; therefore the sample was not fully representative of the population we were trying to target with the instrument. The purposive and convenience samples added further limitations to the generalizability of the findings, as they might have inadvertently excluded some groups of respondents. Given that the qualitative sample recruited post-stroke participants, its criteria might have been too narrow to enable the sample to be generalized to other chronic illnesses, and thus certainly might not reflect the ultimate interest in those with other chronic diseases. However, having considering that specific measures are more sensitive for the disease of interest, while generic measures help to compare health-related quality of life (HRQOL) among different diseases, our measure generated in stage 1 interestingly conveyed no specific description of the illness concerned, but contained general expressions of holistic concern after an episode of chronic illness. These 
findings from using heterogeneous samples with various kinds of chronic diseases have successfully derived a factor model accounting for over $56 \%$ of total explained variance with the established reliability and validity of the HHSQ instrument for expressing generic measures of holistic health. Future research needs to be conducted to test the sensitivity of this generic measure and establish its content validity in assessing the wellbeing of clients with specific diseases of interest.

Using the SF-12 Health Survey in the convergent validity, it fell way short of validating the spiritual components of the HHSQ since SF-12 does not have an explicit spiritual dimension in its measure. Validating the HHSQ in the future might mean administering it along with the scales that will address the specific spiritual dimension. While our findings entailed the discussion of "fate" and "praying to God/gods", which echoed two of the external health loci of control dimensions the belief in chance and the God locus of health control, scales focused on a multidimensional health locus of control might be appropriate for future validation of the HHSQ.

The authors also attempted to evaluate the factor structure of the HHSQ using confirmatory factor analysis in one of the samples in the second stage of the study, but the 8-factor, 45-item HHSQ did not provide a satisfactory fit to the data - the goodness-of-fit indices of $<0.90$ and the value of the root mean square error of approximation (RMSEA) were not within the range of $0.05-0.08$. The study also fell short of recruiting a sufficient number of participants to take part in a two-week test-retest stability check. Although test-retest stability had shown an intra-class coefficient (ICC) of 0.85 , it was based on far too few participants $(n=7)$ to give meaningful stability data. Response biases such as those inherent in the self-reporting survey might be a limitation, hence the possibility of over- and under-reporting biases toward desirable and less acceptable responses respectively should be considered. This study only provides initial psychometric findings on the HHSQ, but future studies are warranted to further validate and improve the scale.

The researchers employed the Classical Test Theory (CTT) approach in analyzing the data. The Item Response Theory (IRT) has advantages over the CTT in estimating health outcomes since it has more vigorous assumptions in data fit and is less sample-dependent [77]. The IRT is also more sensitive in detecting change in health over time [78]. However, the IRT requires a larger sample size (usually over 500) than the CTT (200 to 500 in general) for item parameter estimation [77]. Our study has a sample size of just over 300, so it is difficult to perform an accurate item calibration using the IRT for the existing sample.

\section{Conclusion}

The increased prevalence of chronic diseases is a global health issue. Once an individual has been diagnosed with a chronic disease, healthcare support in different aspects is essential. In this regard, a culturally-sensitive and context-relevant assessment tool and an evaluation of holistic health are central to healthcare practice. This study has taken a step towards exploring the conceptual holistic framework relevant to healthcare in the management of chronic disease, and operationalizing it. The 8-factor, 45-item HHSQ was initially developed in this study - this holistic care measurement might stimulate more studies although the instrument itself might not be mature enough. It appears promising for use in research to assess holistic health status, as it taps into the relational experiences and connectedness within the bio-psycho-social-spiritual dimensions of a person with chronic disease. The HHSQ has the potential to be used in all Chinese-speaking populations with its established culture relevance. While this instrument may contain expressions of well-being that are more emphasized by the Chinese, such as the concept of karma, there are also other more generic items that are shared by non-Chinese respondents, such as feeling confused. It will also be interesting to translate this instrument with confirmed language equivalence to make cross-cultural comparisons of holistic health. The language used in this instrument is derived from the subjective expressions of patients who have experienced living with chronic illness.

\section{Competing interests}

The authors declare that they have no competing interests.

\section{Authors' contributions}

CWC drafted the manuscript, was involved in the psychometric design, analysis and interpretation of the data, revised the manuscript critically, and read and approved the final manuscript. FW led the team in conceptualizing the study idea, contributed to item generation and validity testing, and provided critical comments and input in the composition of the final draft. SY and FS participated in the validation of the questionnaire statements and the coordination of patient interviews, and critically read the final manuscript. All authors read and approved the final manuscript.

\section{Acknowledgements}

This study is supported by Nethersole Institute of Continuing Holistic Health Education (5-ZH64). The research team is also indebted to the support from the clinical team of the study hospitals including Ms Eva Kan, Kit-hoi Chan, Flora Wong, Mei-ha Ip, and Dr Yuk-lun Cheng.

\section{Author details}

${ }^{1}$ School of Nursing, The Hong Kong Polytechnic University, Hung Hom, Kowloon, Hong Kong. ${ }^{2}$ Nethersole Institute of Continuing Holistic Health Education, Room J-7-28, 11 Chuen On Road, Tai Po, NT, Hong Kong.

Received: 19 May 2015 Accepted: 20 January 2016 Received: 19 May 2015 Accepted: 20 January?

\section{References}

1. World Health Organization. World health statistics 2009. Geneva: World Health Organization; 2009

2. Hong Kong Department of Health. Promoting health in Hong Kong. A Strategic Framework for Prevention and Control of Non-communicable Diseases. 2013:Available from http://www.change4health.gov.hk/ 
filemanager/common/image/strategic_framework/promoting_health/ promoting_health_e.pdf. Accessed on 28 June 2013.

3. Census and Statistics Department. Thematic Household Survey Report No. 58, Hong Kong, China SAR 2015: Available from (http://www.statistics.gov. hk/pub/B11302582015XXXXB0100.pdf). Accessed on 23 January 2016.

4. American Heart Association. Heart and stroke statistical update: 2009. Dallas: American Heart Association; 2009.

5. Fisher M, Norrving B. The International Agenda for Stroke. 1st Global Conference on Healthy Lifestyles and Noncommunicable Diseases Control, Moscow, Russia. 2011:Available from http://www.who.int/nmh/ events/moscow_ncds_2011/conference_documents/second_plenary_ norrving_fisher_stroke.pdf. Accessed on 13 June 3.

6. Wang YJ, Cui LY, Ji XM, Dong Q, Zeng JS, Wang YL, et al. The China National Stroke Registry for patients with acute cerebrovascular events: design, rationale, and baseline patient characteristics. Int I Stroke. 2011;6:355-61.

7. Hong Kong Department of Health. Be aware of "brain attack". Non Communicable Dis Watch. 2008:5(5):1-4.

8. United Kingdom of Department of Health. National stroke strategies. London: United Kingdom: Department of Health; 2007.

9. Faircloth CA, Boylstein C, Rittman M, Gubrium JF. Constructing the stroke: sudden-onset narratives of stroke survivors. Qual Health Res. 2005;15:928-41.

10. Green TL, King KM. Experiences of male patients and wife-caregivers in the first year post-discharge following minor stroke: a descriptive qualitative study. Int J Nurs Stud. 2009;46:1194-200.

11. Widar M, Samuelsson L, Karlsson-Tivenius S, Ahlstrom G. Long term pain condition after a stroke. J Rehabil Med. 2002;34:165-70.

12. Clarke P, Black SE. Quality of life following stroke: negotiating disability, identity and resources. J Appl Gerontol. 2005;24:319-36.

13. Lyon BL. Psychological stress and coping: framework for poststroke psychosocial care. Top Stroke Rehabil. 2002;9:1-15.

14. Ostwald SK, Bernal MP, Cron SG, Godwin KM. Stress experienced by stroke survivors and spousal caregivers during first year after discharge from inpatient rehabilitation. Top Stroke Rehabil. 2009;16:93-104.

15. $\mathrm{Ch}^{\prime} \mathrm{Ng} \mathrm{AM}$, French D, Mclean N. Coping with the challenges of recovery from stroke: long term perspectives of stroke support group members. J Health Psychol. 2008;13:1136-46.

16. Arnaert A, Filteau N, Sourial R. Stroke patients in the acute care phase: the role of hope in self healing. Holist Nurs Pract. 2006;20:137-46.

17. Sulmasy DP. A biopsychosocial-spiritual model for the care of patients at the end of life. The Gerontologist. 2002:42(3):24-33.

18. McSherry W. The meaning of spirituality and spiritual care within nursing and health care practice: a study of health care professionals, patients and the public. London: Quay Books; 2007.

19. Patterson EF. The philosophy and physics of holistic health care: spiritual healing as a workable interpretation. J Adv Nurs. 1998;27:287-93.

20. Duncan PW, Zorowitz R, Bates B, Choi JY, Glasberg JJ, Graham GD, et al. Management of adult stroke rehabilitation care: a clinical practice guideline. Stroke. 2005;36:e100-e43.

21. Teasdale TW, Engberg AW. Psychosocial consequences of stroke: a long-term population follow-up. Brain Inj. 2005;19:1049-58.

22. Pajalic Z, Karlsson S, Westergren A. Functioning and subjective health among stroke survivors after discharge from hospital. J Adv Nurs. 2006;54:457-66

23. Dam H, Harhoff M, Andersen PK, Kessing LV. Increased risk of treatment with antidepressants in stroke compared with other chronic illnesss. Int Clin Psychopharmacol. 2007;22:13-29.

24. McSherry W. Making sense of spirituality in nursing and health care practice: an integrative approach. London: Jessica Kingsley Publishers; 2006.

25. American Holistic Nurses' Association \& American Nurses Association. Holistic nursing scope and standards of practice. Silver Spring, MD: American Nurses Association; 2007.

26. Chan CLW, Ho RTH, Fu W, Chow AYM. Turning curses into blessings. J Psychosoc Oncol. 2006;24:15-32

27. Chan CLW, Ng SM, Ho RTH, Chow AYM. East meets west: applying Eastern spirituality in clinical practice. J Clin Nurs. 2006;15:822-32.

28. Shin SR, Eschiti VS. East meets west: a search for Holism in Korean Nursing Practice. J Holist Nurs. 2005;23:356-62.

29. Sun TLC. Themes in Chinese psychology. Singapore: Cengage Learning Asia Pte Ltd; 2008.

30. Hui VKY, Fung HH. Mortality anxiety as a function of intrinsic religiosity and perceived purpose of life. Death Stud. 2009;33:30-50.
31. Bond MH. Chinese values. In: Bond MH, editor. The handbook of Chinese psychology. Hong Kong: Oxford University Press; 1996. p. 208-26.

32. Owolabi MO. Health related quality of life and the seed of life model. J Altern Med Res. 2009;1(4):375-82.

33. Owolabi MO. Psychometric properties of the HRQOLISP-40: A novel, shortened multiculturally valid holistic stroke measure. Neurorehabil Neural Repair. 2010;24(9):814-25.

34. Owolabi MO. Impact of stroke on health-related quality of life in diverse cultures: the Berlin-Ibadan multicenter international study. Health Qual Life Outcomes. 2011;9(81):1-11.

35. Long AF, Mercer $G$, Hughes $K$. Developing a tool to measure holistic practice: a missing dimension in outcomes measurement within complementary therapies. Complemen Ther Med. 2000;8:26-31.

36. Faull K, Hills D. The QE Health Scale (QEHS): assessment of the clinical reliability and validity of a spiritually based holistic health measure. Disabil Rehabil. 2007a;29:701-16.

37. Faull K, Hills D. A spiritually based measure of holistic health for those with physical disabilities: development, and preliminary reliability and validity assessment. Disabil Rehabil. 2007b;29:701-16.

38. Li L, Wang HM, Shen Y. Chinese SF-36 Health Survey: translation, cultural adaptation, validation, and normalisation. J Epidemiol Community Health. 2003:57:259-63.

39. Wagner AK, Gandek B, Aaronson NK, Acquadro C, Alonso J, Apolone G, et al. Cross-cultural comparisons of the content of SF-36 translations across 10 countries: results from the IQOLA project. J Clin Epidemiol. 1998;51(11):925-32.

40. Lange JW. Methodological concerns for non-Hispanic investigators conducting research with Hispanic Americans. Res Nurs Health. 2002;25:411-9.

41. Shin H. Comparison of quality of life measures in Korean menopausal women. Res Nurs Health. 2012;35:383-96.

42. Tseng HM, Lu JFR, Gandek B. Cultural issues in using the SF-36 Health Survey in Asia: results from Taiwan. Health Qual Life Outcomes. 2003;1:72-81.

43. Ware JE, The SCD, MOS. 36-Item Short-Form Health Survey (SF-36): L. Conceptual Framework and Item Selection. Med Care. 1992;30(6):473-83.

44. Portney $L G$, Watkins MP. Foundations of clinical research: applications to practice. Norwalk, CT: Appleton \& Lange; 2000

45. Crum JC, Anthony JC, Bassett SS, Folstein MF. Population-based norms for the Mini-Mental State Examination by age and educational level. J Am Med Assoc. 1993;269:2386-91.

46. Yeung SM, Wong FKY, Mok E. Holistic concerns of Chinese stroke survivors during hospitalization and in transition to home. J Adv Nurs. 2011;67:2394-405.

47. Polit DF, Beck CT. Nursing research: generating and assessing evidence for nursing practice. Philadelphia: Lippincott; 2012.

48. Nunnally JC, Bernstein $\mathbb{H}$. Psychometric Theory. 3rd ed. New York: McGraw-Hill; 1994.

49. Ware JE, Kosinski M, Keller SD. A 12-item Short-Form Health Survey: construction of scales and preliminary tests of reliability and validity. Med Care. 1996;34(3):220-33.

50. Lam CLK, Tse EYY, Gandek B. Is the standard SF-12 Health Survey valid and equivalent for a Chinese population? Qual Life Res. 2005;14(2):539-47.

51. Lam ETP, Lam CLK, Fong DYT, Huang WW. Is the SF-12 version 2 Health Survey a valid and equivalent substitute for the SF-36 version 2 Health Survey for the Chinese? J Evaluat Clin Pract. 2013;19(1):200-208.

52. Tabachnick BG, Fidell LS. Using multivariate statistics. Boston, MA: Allyn and Bacon; 2007.

53. Hair JF, Anderson RE, Tatham RL, Black WC. Multivariate data analysis. Upper Saddle River, NJ: Prentice-Hall; 2010

54. Kline P. Psychometrics primer. London: Free Association Books; 2000.

55. Dyson J, Cobb M, Forman D. The meaning of spirituality: a literature review. J Adv Nurs. 1997:26:1183-8.

56. Carson VB. Spiritual dimensions of nursing practice. Philadelphia: W. B. Saunders Company; 1989.

57. Lemmer CM. Recognizing and caring for spiritual needs of clients. J Holist Nurs. 2005:23:310-22

58. Mok E, Wong F, Wong D. The meaning of spirituality and spiritual care among the Hong Kong Chinese terminal ill. J Adv Nurs. 2010;66:360-70.

59. Stoll Rl. Spirituality and chronic illness. In: Carson VB, editor. Spiritual dimensions of nursing practice. Philadelphia, PA: W. B. Saunders Company: 1989. p. $180-216$. 
60. Schillebeeckx E. God among us: the Gospel proclaimed. London: SCM Press; 1983.

61. See M-DC. Aquinas and His Role in Theology, Paul Philibert, trans. Minnesota: Order of St. Benedict; 2002.

62. Selvam SG, Poulsom M. Now and Hereafter. The Psychology of Hope from the Perspective of Religion. J Dharma. 2012;37(4):393-410.

63. Cope-Kasten V. Meeting Chinese philosophy. In: Giskin H, Walsh B, editors. An introduction to Chinese culture through the family. New York: State University of New York Press; 2001. p. 41-57.

64. Bond MH, Hwang KK. The social psychology of Chinese people. In: Bond MH editor. The psychology of the Chinese people. Hong Kong: Oxford University Press; 1986. p. 213-66.

65. Bowes S, Lowes L, Warner J, Gregory JW. Chronic sorrow in parents of children with type 1 diabetes. J Adv Nurs. 2009;65:992-1000.

66. Mok E, Lai CKY, Wong FLF, Wan P. Living with early-stage dementia: the perspective of older Chinese people. J Adv Nurs. 2007;59:591-600.

67. Siu O, Spector PE, Cooper CL. A three-phrase study to develop and validate a Chinese coping strategy scale in Greater China. Personal Individ Differ. 2006;41:537-48.

68. Floyd F, Widaman KF. Factor analysis in the development and refinement of clinical assessment instruments. Psychol Assess. 1995;7:286-99.

69. Polit-O'Hara D. Data analysis statistics for nursing research. Stanford, CA: Appleton Lange; 1996.

70. Kline P. An easy guide to factor analysis. New York: Routledge; 1997.

71. Nunnally JC. Psychometric theory. New York: McGraw-Hill Book Company; 1978.

72. Streiner DL, Norman GR. Health measurement scales: a practical guide to their development and use. New York: Oxford University Press; 1995.

73. Wagner J, Lacey K, Chyun D, Abbott G. Development of a questionnaire to measure heart disease risk knowledge in people with diabetes: the Heart Disease Fact Questionnaire. Patient Educ Couns. 2005;58(1):82-7. doi:10. 1016/j.pec.2004.07.004

74. Gorsuch RL. Factor analysis. New Jersey: Lawrence Erlbaum Associates, Publishers; 1983

75. Briggs SR, Cheek JM. The role of factor analysis in the development and evaluation of personality scales. J Pers. 1986;54:106-48.

76. Chung LYF, Wong FKY, Chan MF. Relationship of nurses' spirituality to their understanding and practice of spiritual care. J Adv Nurs. 2007;58:158-70.

77. Hambleton RK, Jones RW. Comparison of classical test theory and item response theory and their applications to test development. Educ Measure. 1993;12:38-47.

78. Hays RD, Morales LS, Reise SP. Item response theory and health outcomes measurement in the 21st century. Med Care. 2000;38:|128-42.

\section{Submit your next manuscript to BioMed Central and we will help you at every step:}

- We accept pre-submission inquiries

- Our selector tool helps you to find the most relevant journal

- We provide round the clock customer support

- Convenient online submission

- Thorough peer review

- Inclusion in PubMed and all major indexing services

- Maximum visibility for your research

Submit your manuscript at www.biomedcentral.com/submit

) Biomed Central 